\title{
Use of Magnetic Resonance Microscopy for the Nondestructive Observation of Xylem Cavitation Caused by Pine Wilt Disease
}

\author{
Shin Utsuzawa, Kenji Fukuda, and Daisuke Sakaue
}

First author: Institute of Applied Physics, University of Tsukuba, Ibaraki, Japan and MR Technology, Inc., Tsukuba, Japan; second author: Laboratory of Biosphere Functions, Institute of Environmental Studies, Graduate School of Frontier Sciences, the University of Tokyo, 113-8657, Japan; and third author: Experimental Station at Tanashi, University Forests, the University of Tokyo, Japan. Accepted for publication 27 February 2005.

\begin{abstract}
Utsuzawa, S., Fukuda, K., and Sakaue, D. 2005. Use of magnetic resonance microscopy for the nondestructive observation of xylem cavitation caused by pine wilt disease. Phytopathology 95:737-743.

The development of xylem cavitation caused by pine wilt disease was visualized nondestructively with a compact magnetic resonance (MR) microscope system. A $\mathrm{T}_{1}$-weighted spin-echo sequence clearly visualized

the pinewood nematode (Bursaphelenchus xylophilus), and enlarged gradually over several days. After 11 to 18 days, cavitated areas rapidly increased in size, fused, and reached the cambium. This drastic expansion in cavitation coincided with and appeared to explain the sudden wilting of the seedlings. The development of cavitation observed through MR microscopy corresponded well with previous descriptions of disease progression.
\end{abstract} the water-filled xylem of Japanese black pine (Pinus thunbergii) as white zones, whereas cavitated xylem was represented as dark areas. Cavitated areas in the xylem were first observed 6 to 9 days after inoculation with
Additional keywords: embolism, magnetic resonance imaging, water transport.
Vascular wilting diseases such as Dutch elm disease and oak wilt are fatal to trees and can cause serious problems in forests and urban areas. Pine wilt disease, induced by the pinewood nematode Bursaphelenchus xylophilus, is the most serious tree epidemic in Japan and eastern Asia. Over the last 30 years in Japan, this disease has annually killed about $1,000,000 \mathrm{~m}^{3}$ of pine trees (2).

Pine wilt disease has been intensively studied for some decades, and the development of disease symptoms has been divided into two stages: early and advanced (3). In the early stage, nematodes migrate through cortical and xylem resin canals in pine stems. They induce cavitation (breaking of the water column in tracheids), embolism (filling of the tracheids with gas), and occlusion of the tracheids with resin; in transverse sections of the stem, the affected tissues appear as dry patches. In the advanced stage, the nematodes multiply and destroy the cambium, which induces dysfunction of water conduction in the entire xylem and causes water potential, transpiration, and photosynthesis to rapidly decrease. Consequently, the needles wilt and the tree dies suddenly.

The key to understanding pine wilt symptom development may be to understand the shift from the early to the advanced stage. But understanding this shift has been difficult because it occurs so rapidly. Xylem cavitation and other histological changes, including the denaturation of ray parenchyma and the destruction of the cambium, have been observed by destructive methods such as sectioning and staining of the stem. Unfortunately, destructive methods make it impossible to continuously observe symptom development in the same individual. Although symptom development might be inferred by destructive sampling of many different trees, the timing of symptom development among individual trees

Corresponding author: K. Fukuda; E-mail address: fukuda@k.u-tokyo.ac.jp

DOI: 10.1094/PHYTO-95-0737

(c) 2005 The American Phytopathological Society often varies considerably. Some trees exhibit symptoms within 2 to 3 weeks after nematode infection, whereas others do not show symptoms for several months or years. Location of symptoms also varies. In some trees, symptoms are restricted to the area near the inoculation site, but in others, symptoms may appear in upper branches or in the roots. Thus, a nondestructive method for continuous observation of the same individual tree or seedling would be very useful.

Nondestructive methods for the diagnosis of human diseases are common and include X-ray computed tomography (CT), magnetic resonance imaging (MRI), and ultrasonic tomography. Such clinical technology is theoretically applicable to plants, although considerable rearrangement of the apparatus would be necessary because the organism size and the required resolution differ greatly between humans and plants. Recently, MRI has been used as a noninvasive way to study the water status of plants $(1,5$, 9,10,13,15). Ikeda (8) and Kuroda et al. (11) have demonstrated that cavitation in the xylem of pine trees inoculated with the pinewood nematode can be visualized using clinical MRI systems. But because the resolution of clinical MRI systems is insufficient to detect small cavitation areas in seedlings, clinical MRI can only be used to observe large cavitated areas in saplings and trunks of downed trees.

MR microscopes with super-conducting cryomagnets $(1,13)$ have a sufficiently fine resolution $(<100 \mu \mathrm{m})$ to reveal small areas of cavitation; these microscopes, however, cannot accommodate a whole pine seedling with its branches spread. Moreover, because these microscopes require large spaces with high ceilings for the super-conducting magnet and procure high coolant costs, most botanists and plant pathologists are unable to sustain their use over the long term. MR microscopes with noncooled electromagnets avoid these problems and have been used to observe xylem water (15) with high resolution.

In this study, we constructed a compact MR microscope system with an open permanent magnet to nondestructively observe tree seedlings for 25 days. This system was used to investigate the development of cavitation caused by pine wilt disease in situ. 


\section{MATERIALS AND METHODS}

The experiment was performed with 3-year-old Japanese black pine (Pinus thunbergii Parl.) seedlings. The pine seedlings, which were in plastic pots containing 3 liters of soil, were grown in the nursery at the Experimental Station at Tanashi, the University Forests, Graduate School of Agricultural and Life Sciences, the University of Tokyo.

A virulent isolate (S-10) of the pinewood nematode was cultured at $25^{\circ} \mathrm{C}$ for 2 weeks on mycelia of Botrytis cinerea grown on potato dextrose agar plates. Nematodes were recovered from the cultures with Baermann funnels. The xylem of the current shoot of the main stem was inoculated with 10,000 nematodes suspended in $0.01 \mathrm{ml}$ of water through a notch cut with a razor blade. The inoculation wound was wrapped with polyvinyl tape (Fig. 1A). Three seedlings were inoculated on different days, and each was placed in the MR microscope for the duration of its experimental period. The inoculations occurred on 24 October 2003 (seedling 1), 1 March 2004 (seedling 2), and 12 August 2004 (seedling 3). A control seedling was injected with water but not nematodes on 14 November 2004 and was observed with the MR microscope daily for 24 days.

For the MR measurements, an eight-turn solenoid RF (radio frequency, an electromagnetic wave with a frequency in the same range as radiowaves) coil (10-mm inner diameter) was wrapped around the 1-year-old plant stem, below the inoculation point and $15 \mathrm{~cm}$ above the soil surface (Fig. 1A). The coil, which could be opened or folded, was installed in an RF shield box made of 0.5 -mm-thick brass plates.

The compact MR microscope system used in this study (Fig. 1B) consisted of a four-column permanent magnet (Neomax Co. Ltd., Japan; 1.0-T (tesla) field strength, 6-cm air gap) and a portable MRI console (MR Technology, Inc., Japan). A pair of flat gradient coils was fixed onto the pole pieces of the magnet. The

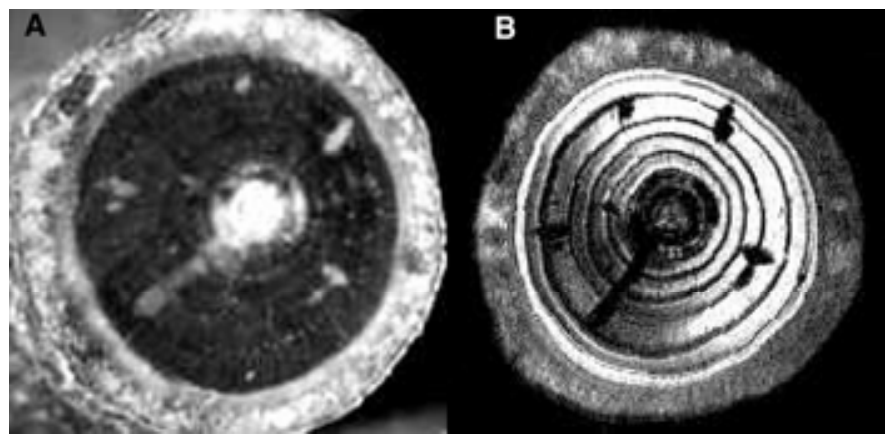

Fig. 2. Transverse sections of a Japanese red pine branch naturally infected with pinewood nematode. A, The cut surface of the branch after absorption of acid fuchsin solution. Cavitated xylem appears as light patches. B, Spin-echo $\mathrm{T}_{1}$-weighted magnetic resonance image (transverse-slice) at the same position but before the branch was cut. Repetition time (TR) $=500 \mathrm{~ms}$, echo time $(\mathrm{TE})=22 \mathrm{~ms}, 256 \times 256$ pixels. Cavitated xylem appears as dark patches. Branch diameter $10 \mathrm{~mm}$.

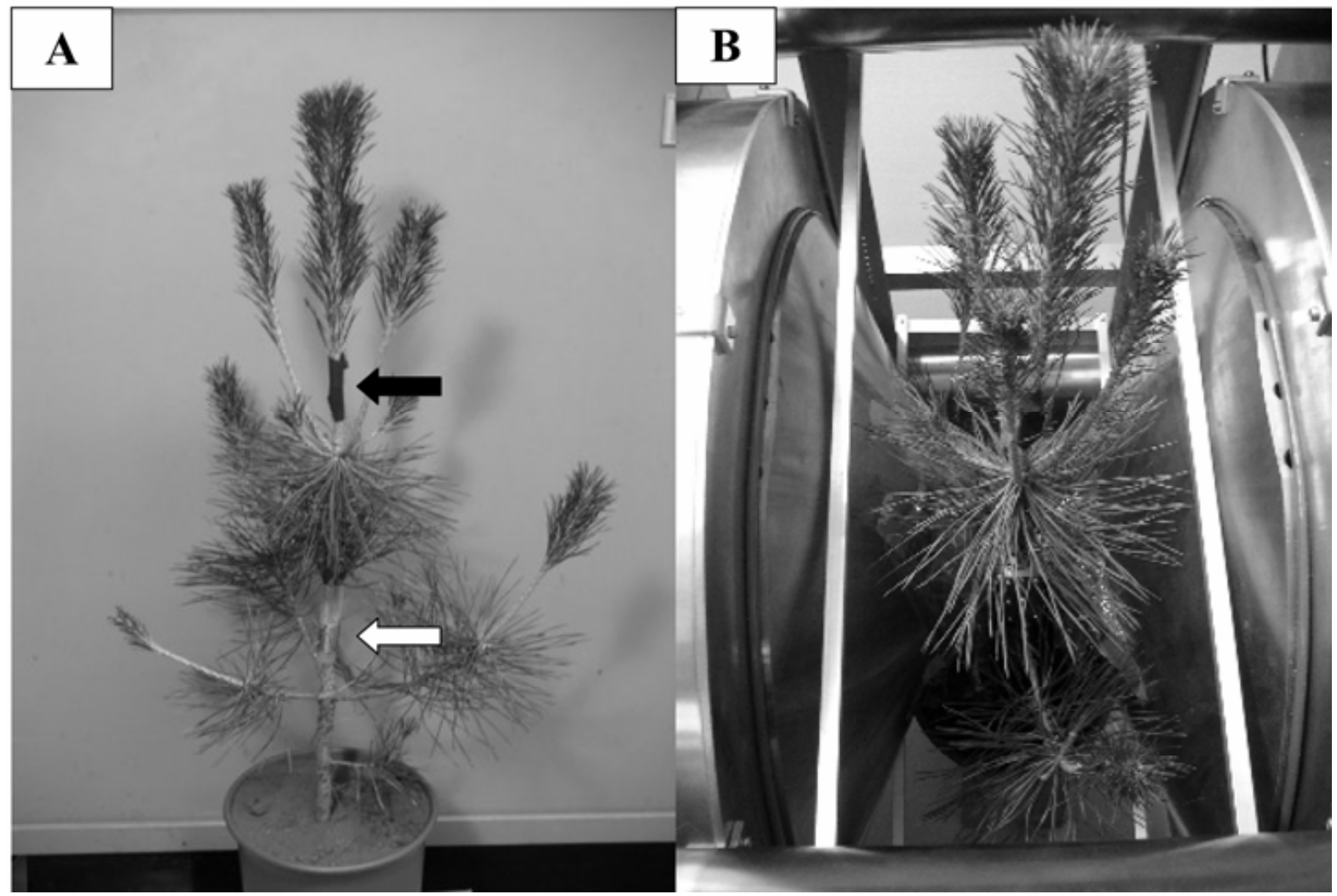

Fig. 1. Japanese black pine seedling inoculated with the pinewood nematode and positioned in the magnetic resonance (MR) microscope system. A, The main current-year shoot was inoculated with nematodes and the inoculated area was taped (black arrow). Before the seedling was placed in the MR microscope, an RF (radio frequency) coil was formed around the area to be observed (white arrow), which had been wrapped with plastic sheeting and which was located on the 1-year-old stem. Seedling height was $60 \mathrm{~cm}$. B, MR microscope system with a pair of cone-shaped permanent magnets on either side of the inoculated seedling. The horizontal field of $1.0 \mathrm{~T}$ was provided by a permanent magnet, which had an air gap of $6 \mathrm{~cm}$ and which was equipped with a pair of planer gradient coil sets. The diameter and the widest distance between the two magnetic cones were 35.5 and $28 \mathrm{~cm}$, respectively. 


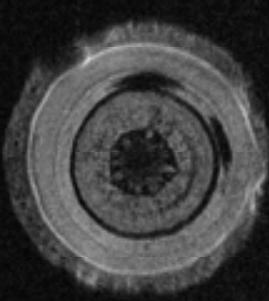

day 0

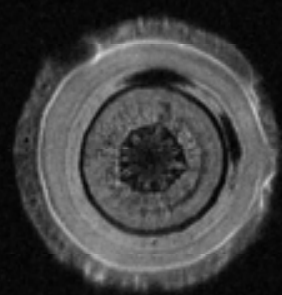

day 8

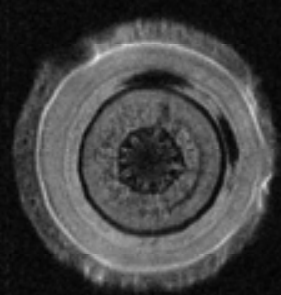

day 16

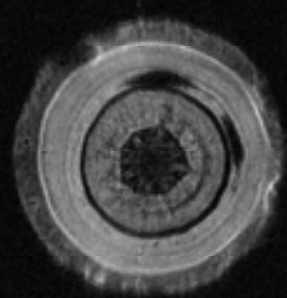

day 24

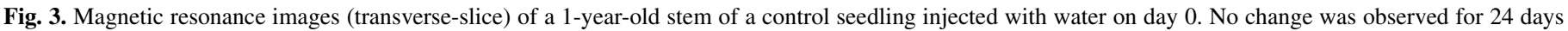
after water injection. Stem diameter $9 \mathrm{~mm}$.

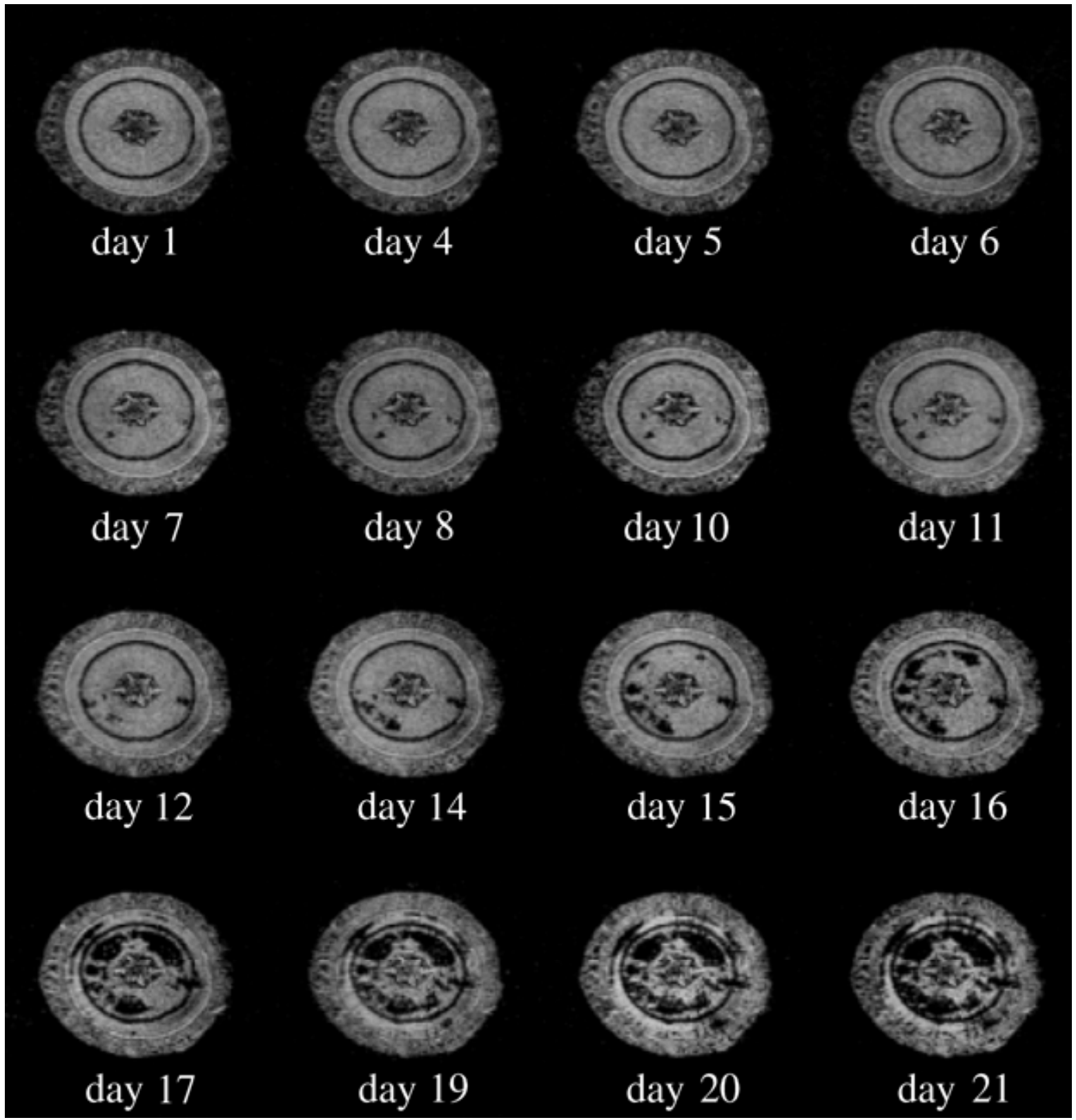

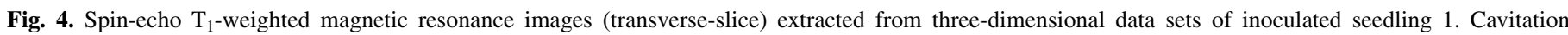
increased greatly from day 14 to day 17 . Repetition time $(\mathrm{TR})=500 \mathrm{~ms}$, echo time $(\mathrm{TE})=10 \mathrm{~ms}, 128 \times 128$ pixels. Stem diameter $8 \mathrm{~mm}$. 
pine seedling was placed between them, and nondestructive observations were conducted throughout the experimental periods. A three-dimensional image matrix of $128 \times 128 \times 256$ pixels with a view field of $19.2 \mathrm{~mm}^{3}$ (for seedling 1) or a transverse twodimensional image of $256 \times 256$ pixels (for seedlings 2, 3, and control) was obtained daily after the inoculation with nematodes. Illumination and water were supplied to the seedlings from the open top and both sides of the magnet. Seedlings received about $1,000 \mu \mathrm{mol} / \mathrm{m}^{2} / \mathrm{s}$ for $4 \mathrm{~h}$ on sunny days and auxiliary light (SunLamp 40W, Kyokko Denki Kogyo Co. Ltd., Tokyo) during sunless hours of daytime. When soil was dry (every 2 or 3 days), about $500 \mathrm{ml}$ of water was added to the pot.

A preliminary study had been conducted to determine appropriate MR parameters and to determine whether our MR microscope revealed xylem cavitation in pine wilt disease. Four branches were collected from a Japanese red pine ( $P$. densiflora) tree naturally infected with pinewood nematode and located on the campus of the University of Tsukuba. MR transverse slice images of the branches were acquired with a range of TE and TR values: $\mathrm{TE}$, or echo time, represents the time in milliseconds between the excitation pulse and the peak of the echo signal in applied pulse sequences; TR, or repetition time, is the time between successive excitation pulses. The values of both TR and TE greatly affect image contrast, and TR is a major factor in total scan time. Optimum MR images were obtained with a TR of $500 \mathrm{~ms}$ and TE of 10 or $22 \mathrm{~ms}$, and these values were used throughout the study.

The preliminary study also confirmed that our MR observations of intact wood corresponded well with direct observation of stained and cut wood (Fig. 2). Previous reports have demonstrated that, when pine wilt-affected wood is stained by acid fuchsin, cut, and directly examined, the conducting xylem (water filled) appears dark in a black and white image (red in a color image) whereas cavitated xylem appears white $(3,4,14)$. In one of the four stained and cut branches, five white patches of cavitated xylem were apparent (Fig. 2A). In the MR image taken from the same branch and position before the branch was stained and cut, the same patches appeared black rather than white (Fig. 2B). Similar correspondences were obtained with the three other pine wilt-affected branches (data not shown). This observation, that cavitated tissue appears dark and water-filled tissue appears light in a $\mathrm{T}_{1}$-weighted MR image, agrees with other reports $(1,5,9,11)$. The observation also agrees with previous MRI studies of pine saplings inoculated with pinewood nematode $(8,11)$. These previous studies, however, did not check the water content of the tracheids (by comparing data from MRI with data from stained and cut tissue), and their MRI apparatus was not suitable for continuous observation of the same plant.

\section{RESULTS AND DISCUSSION}

In the control seedling, we observed two dark areas in the current-year earlywood, which had been present before the inoculation with water (Fig. 3). These areas were assumed to be compression wood often seen in this species. After inoculation with water, no change in the MR image was detected, i.e., the control did not exhibit cavitation (Fig. 3). In seedling 1, dark areas of the xylem, which represent cavitations caused by the pinewood nematode, were first observed on day 7 as four dark spots in the earlywood of the last year (Fig. 4). The number and area of cavitations grew gradually until day 14 ; this period was regarded as the early stage. On day 16, cavitation expanded drastically and occupied most of the xylem. Thus, the advanced stage began in seedling 1 between day 14 and 16 .

After seedling 1 wilted, the last MR image on day 21 was compared with the actual cut surface from the same position on day 30 (Fig. 5). Cavitation in the cut surface could not be observed clearly because the xylem was occluded with resinous compounds that prevented absorption of the dye. The embolized xylem, however, was observed as bright areas that showed a pattern similar to the dark areas of xylem in the MR image. Differences in the exact shape and size of cavitated areas revealed by MR microscopy and destructive sampling may have occurred because the observations were from different days or because the two slices were taken from a slightly different position. In both the MR image and the cut surface, part of the stem xylem contained water in the tracheids even though the seedling had wilted. This occurs when the upper part of the plant wilts before embolism occurs in the stem xylem and water remains in the occluded xylem. Such heterogeneous symptoms can develop under
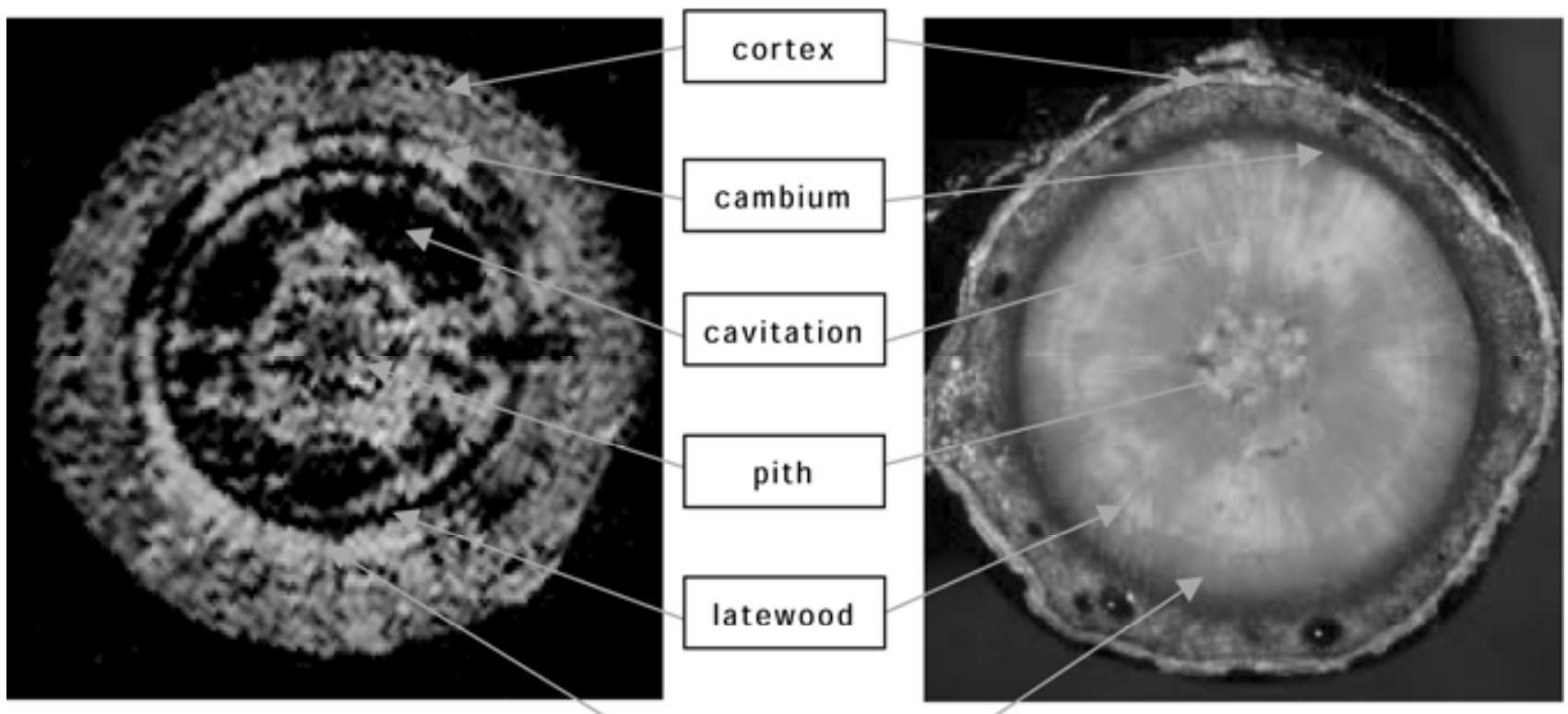

water- and resin-filled xylem

Fig. 5. Spin-echo $T_{1}$-weighted magnetic resonance image of inoculated seedling 1 on day 21 (left) and cut surface at the same position on day 30 (right). Stem diameter $8 \mathrm{~mm}$. 
the lower temperatures in autumn (3). In our study, the upper shoots and branches wilted without yellowing of the needles before day 16, whereas some lower branches remained fresh through day 30 .

Cavitation development in seedling 1 was quantified (Fig. 6). Cavitation was first observed on day 7 , but the area was very small. The cavitated area remained small until day 13, but increased greatly from day 13 to day 17 (Fig. 6A). In the early stage, the cavitated areas were located in last year's xylem and were small in area and number. In the initial phase of the advanced stage from day 14 to 16 , many new patches occurred nearly simultaneously, and during these 3 days, the cavitations expanded rapidly and fused. On day 17, cavitations expanded beyond the last annual ring into the current-year xylem, and many new small cavitations occurred along the cambium (Fig. 6B).

Our MR observations have indicated that the expansion of cavitation in the advanced stage is very rapid, occurring within only 3 days. Such a rapid process would not have been observable without this continuous and nondestructive method. The rapidly expanding cavitation appears to correspond to the process of "run-away embolism," which has been hypothesized from acoustic emissions (8). And the development of small cavitations in and near the cambium agrees with previous anatomical observations of cambium destruction by multiplying nematodes in the advanced stage of pine wilt disease $(3,4,6,12,14)$.

Cavitation development in seedlings 2 and 3 followed a pattern similar to that in seedling 1 (Figs. 7 to 9). Cavitation was first observed on day 9 in seedling 2 and on day 7 in seedling 3 . The areas of cavitation expanded dramatically from day 9 to 16 in seedling 2, and from day 19 to 23 in seedling 3. In seedling 2, the rate of expansion decreased from day 11 to 13 but increased thereafter; by day 16 , the cavitation had spread to the cambium. In seedlings 2 and 3, the entire xylem became cavitated (Fig. 7, day 16; Fig. 8, day 24), and the xylem was confirmed to be dry by destructive observations after wilting of seedling 2 (Fig. 7, day 21)

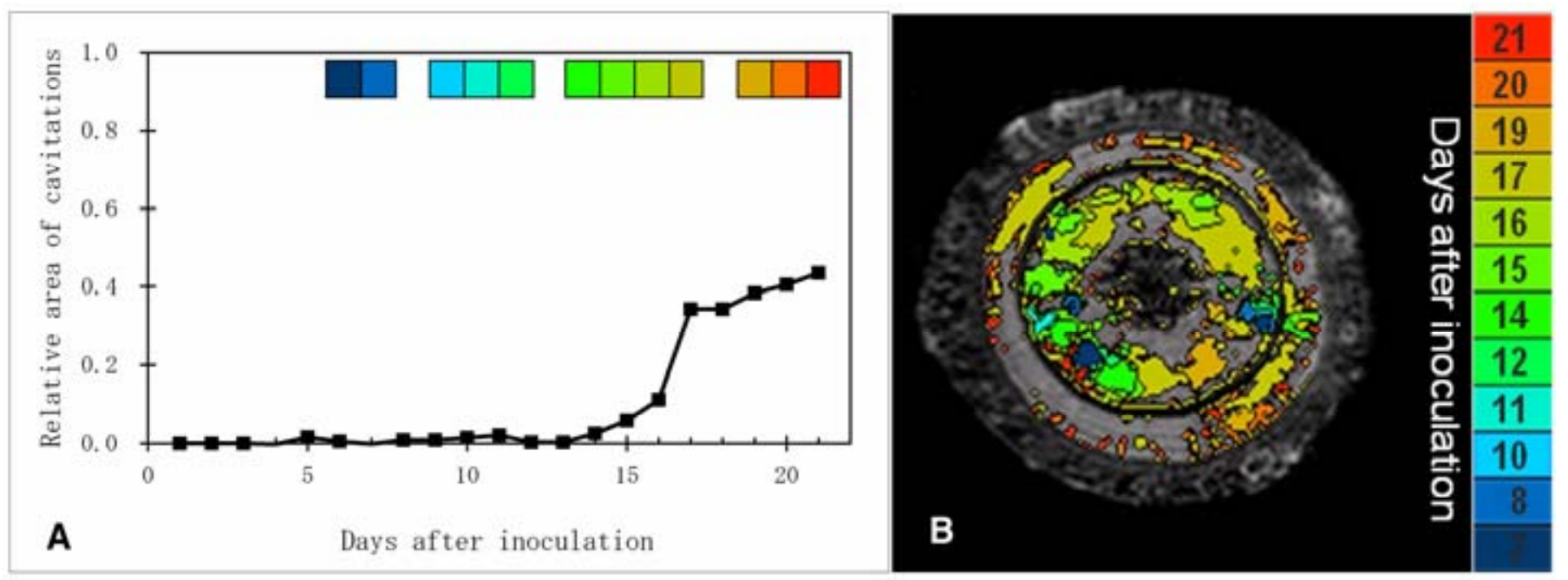

Fig. 6. Cavitation development in the stem of pine seedling 1. A, Proportion of the xylem that was cavitated. B, New cavitations observed each day. The color code on right indicates when cavitation appeared.

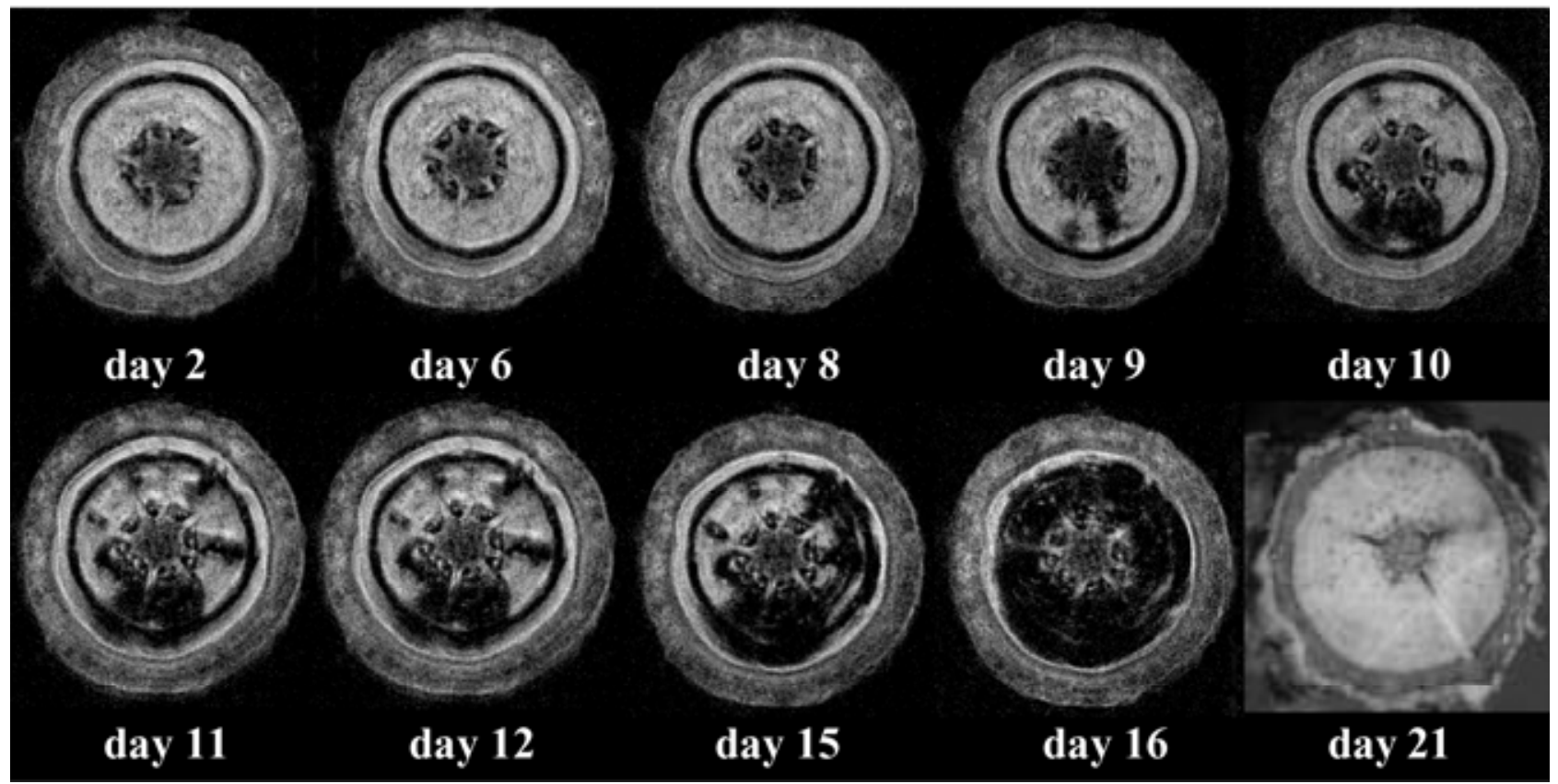

Fig. 7. Spin-echo $T_{1}$-weighted magnetic resonance images (transverse-slice) of inoculated seedling 2 from 2 to 16 days after inoculation and the cut surface at the same position on day 21 . Repetition time $(\mathrm{TR})=500 \mathrm{~ms}$, echo time $(\mathrm{TE})=22 \mathrm{~ms}, 256 \times 256$ pixels. Stem diameter $7 \mathrm{~mm}$. 
and seedling 3 (data not shown). The yellowing of old needles, which is the characteristic symptom of the advanced stage (3), coincided with the rapid expansion of cavitation.

In summary, the three inoculated seedlings but not the control seedling showed cavitation in the xylem when examined with an MR microscope. Cavitation was limited to the inner xylem for about 10 days after infection; during this early stage, the number of cavitated patches and the area of cavitation increased only gradually. The cavitated area then rapidly enlarged and reached the cambium within several days in the advanced stage. This symptom development in the early and advanced stage, which was hypothesized from anatomical observations of multiple plants $(3,14)$, was clearly confirmed by our nondestructive observations of the same individuals using MR microscopy.
To our knowledge, this is the first report of continuous observations of internal pathological changes caused by a plant disease. Our results demonstrate that cavitation caused by a wilting disease can be continuously monitored with MR microscopy. In this study, only one plant was monitored at a time, because keeping one plant immobilized in the MR microscope facilitated the repeated acquisition of images from the same stem position. When longer monitoring periods are required, it may be desirable to monitor several plants in parallel during the same period with one MR microscope. This can be achieved by attaching a marker such as a water-filled capillary to the stem; the water-filled capillary would appear as a bright spot in the MR image and would thus indicate the exact position to be observed.

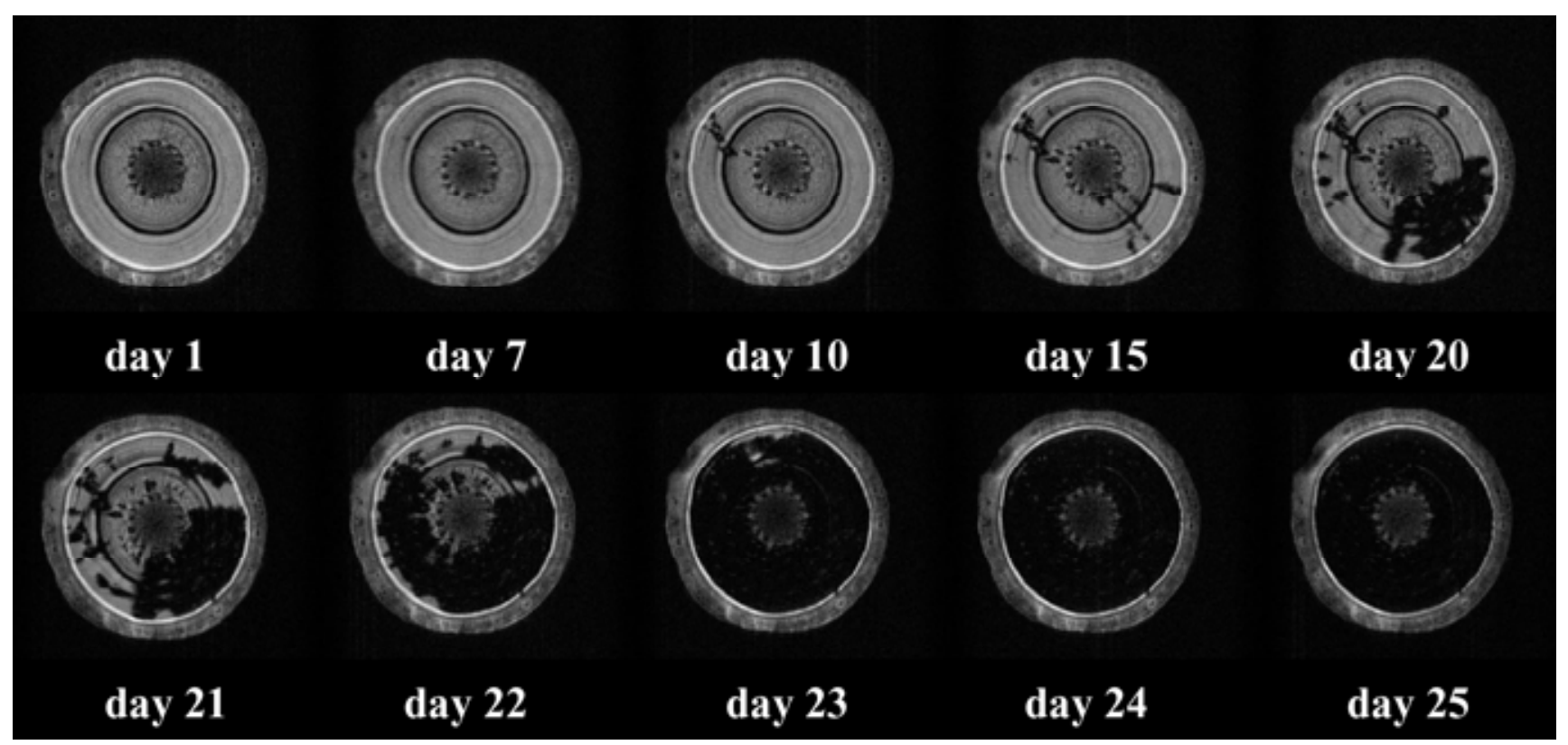

Fig. 8. Spin-echo $T_{1}$-weighted magnetic resonance images (transverse-slice) of inoculated seedling 3 from 1 to 25 days after inoculation. Repetition time (TR) = $500 \mathrm{~ms}$, echo time $(\mathrm{TE})=22 \mathrm{~ms}, 256 \times 256$ pixels. Stem diameter $9 \mathrm{~mm}$.

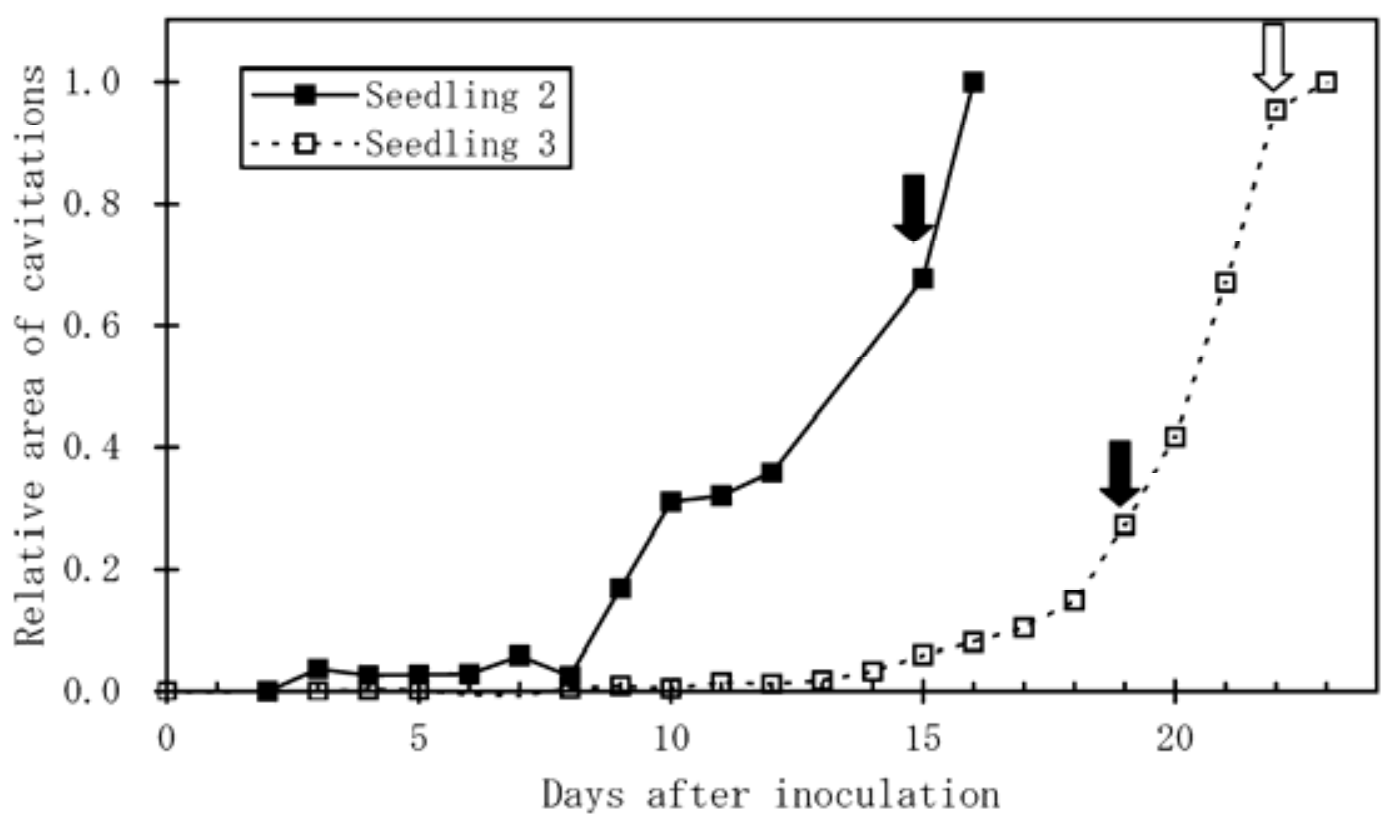

Fig. 9. Cavitation development in seedlings 2 and 3 as revealed by magnetic resonance images (transverse-slice). The $y$ axis refers to the proportion of the xylem that was cavitated. Image data are missing from seedling 2 on days $0,1,13$, and 14 . Solid and open arrows indicate the days when yellowing of old needles and wilting of current needles, respectively, were first observed. 


\section{ACKNOWLEDGMENTS}

We thank T. Haishi (MR Technology, Inc., Japan) and K. Kose (University of Tsukuba, Japan) for their support in constructing and testing the apparatus.

\section{LITERATURE CITED}

1. Clearwater, M. J., and Clark, C. J. 2003. In vivo magnetic resonance imaging of xylem vessel contents in woody lianas. Plant Cell Environ. 26:1205-1214.

2. Forestry Agency. 2004. Annual Report on Trends of Forest and ForestryFiscal Year 2003. (in Japanese) Forestry Agency, The Ministry of Agriculture, Forestry and Fisheries of Japan.

3. Fukuda, K. 1997. Physiological process of the symptom development and resistance mechanism in pine wilt disease. J. For. Res. 2:171-181.

4. Fukuda, K., Hogetsu, T., and Suzuki, K. 1992. Cavitation and cytological changes in xylem of pine seedlings inoculated with virulent and avirulent isolates of Bursaphelenchus xylophilus. J. Jpn. For. Soc. 74:28-299.

5. Holbrook, N. M., Ahrens, E. T., Burns, M. J., and Zwieniecki, M. A. 2001. In vivo observation of cavitation and embolism repair using magnetic resonance imaging. Plant Physiol. 126:27-31.

6. Ichihara, Y. 2000. Pathway of pinewood nematodes. FFPRI Tohoku Br. News 462:1-4.
7. Ikeda, T. 1996. Xylem dysfunction in Bursaphelenchus xylophilusinfected Pinus thubergii in relation to xylem cavitation and water status. Ann. Phytopathol. Soc. Jpn. 62:554-558.

8. Ikeda T. 2002. Ascent of tree sap. Pages 181-188 in: Environmental Tree Physiology. H. Nagata and S. Sasaki, eds. Bun-eido Publ., Tokyo.

9. Ishida, N., Koizumi, M., and Kano, H. 2000. The NMR microscope: A unique and promising tool for plant science. Ann. Bot. 86:259-278.

10. Johnson, G. A., Brown, J., and Kramer, P. J. 1987. Magnetic resonance microscopy of changes in water content in stems of transpiring plants. Proc. Natl. Acad. Sci. 84:2752-2755.

11. Kuroda, K., Kanbara, Y., Inoue, T., and Ogawa, A. 2002. Analysis of NMR-CT images to detect the xylem dysfunction and lesions in tree trunks. IAWA J. 23:469-470.

12. Myers, R. F. 1986. Cambium destruction in conifers caused by pinewood nematodes. J. Nematol. 18:398-402.

13. Rokitta, M., Peuke, A. D., Zimmermann, U., and Haase, A. 1999. Dynamic studies of phloem and xylem flow in fully differentiated plants by fast nuclear-magnetic-resonance microimaging. Protoplasma 209:126131.

14. Sasaki, S., Odani, K., Nishiyama, Y., and Hayashi, Y. 1984. Development and recovery of pine wilt disease studied by tracing the ascent of sap flow marked with water soluble stains. J. Jpn. For. Soc. 66:141-148.

15. Scheenen, T., Heemskerk, A., de Jager, A., Vergeldt, F., and van As, H. 2002. Functional imaging of plants: A nuclear magnetic resonance study of a cucumber plant. Biophys. J. 82:481-492. 